This is a self-archived version of an original article. This version may differ from the original in pagination and typographic details.

Author(s): Zhu, Yongjie; Liu, Jia; Ristaniemi, Tapani; Cong, Fengyu

Title: Distinct Patterns of Functional Connectivity During the Comprehension of Natural, Narrative Speech

Year: 2020

Version: Accepted version (Final draft)

Copyright: @ World Scientific Publishing Company 2020

Rights: In Copyright

Rights url: http://rightsstatements.org/page/InC/1.0/?language=en

Please cite the original version:

Zhu, Y., Liu, J., Ristaniemi, T., \& Cong, F. (2020). Distinct Patterns of Functional Connectivity During the Comprehension of Natural, Narrative Speech. International Journal of Neural Systems, 30(3), Article 2050007. https://doi.org/10.1142/S0129065720500070 


\title{
DISTINCT PATTERNS OF FUNCTIONAL CONNECTIVITY DURING THE COMPREHENSION OF NATURAL, NARRATIVE SPEECH
}

\author{
YONGJIE ZHU \\ School of Biomedical Engineering, Faculty of Electronic and Electrical Engineering \\ Dalian University of Technology, 116024, Dalian, China \\ Faculty of Information Technology \\ University of Jyväskylä, 40014, Jyväskylä, Finland \\ E-mail: yongjie.zhu@foxmail.com \\ JIA LIU \\ Faculty of Information Technology \\ University of Jyväskylä, 40014, Jyväskylä, Finland \\ E-mail: jialiu15@foxmail.com \\ TAPANI RISTANIEMI \\ Faculty of Information Technology \\ University of Jyväskylä, 40014, Jyväskylä, Finland \\ E-mail: tapani.ristaniemi@jyu.fi \\ FENGYU CONG* \\ School of Biomedical Engineering, Faculty of Electronic and Electrical Engineering \\ Dalian University of Technology, 116024, Dalian, China \\ Faculty of Information Technology \\ University of Jyväskylä, 40014, Jyväskylä, Finland \\ E-mail: cong@dlut.edu.cn
}

\begin{abstract}
Recent continuous task studies, such as narrative speech comprehension, show that fluctuations in brain functional connectivity (FC) are altered and enhanced compared to the resting-state. Here, we characterized the fluctuations in FC during comprehension of speech and time-reversed speech conditions. The correlations of Hilbert envelope of source-level EEG were used to quantify FC between spatially separate brain regions. A symmetric multivariate leakage correction was applied to address the signal leakage issue before calculating FC. The dynamic FC was estimated based on a sliding time window. Then, principal component analysis (PCA) was performed on individually concatenated and temporally concatenated FC matrices to identify FC patterns. We observed that the mode of FC induced by speech comprehension can be characterized with a single principal component. The condition-specific FC demonstrated decreased correlations between frontal and parietal brain regions and increased correlations between frontal and temporal brain regions. The fluctuations of the condition-specific FC characterized by a shorter time demonstrated that dynamic FC also exhibited condition-specificity over time. The FC is dynamically reorganized and FC dynamic pattern varies along a single mode of variation during speech comprehension. The proposed analysis framework seems valuable for studying the reorganization of brain networks during continuous task experiments.
\end{abstract}

Keywords: Reorganization; functional connectivity; naturalistic speech; speech comprehension; natural paradigms.

\section{Introduction}

During real-life experiences (e.g., watching a movie or listening to a speech), it is necessary to continuously integrate and parse information. ${ }^{1,2}$ Previous studies have identified a group of high-order brain regions, including the temporal parietal junction, posterior cingulate cortex, temporal pole, and medial prefrontal cortex, which can

\footnotetext{
* Corresponding author.
} 
accumulate and integrate information during comprehension of a narrative story. ${ }^{3,4}$ Although the neural correlation of local information processing has been well investigated in previous studies, integrating information at the whole-brain level may also be critical to understanding brain functions. ${ }^{5,} 6$ Advances in methodology and brain imaging technology have enabled us to examine how the brain mediates information flow in large-scale functional networks during continuous task execution. $^{5,7-10}$

Function connectivity (FC), based on statistical interdependencies between signals recorded using neuroimaging technology, ${ }^{11-14}$ is a widely-used approach to describe the large-scale configuration of brain functional activity. ${ }^{15-18} \mathrm{FC}$ modes provide fingerprints for the organization of functional brain networks during resting state ${ }^{19-21}$ and continuous task performance. ${ }^{22-25}$ Recent studies have demonstrated that there is a robust relationship between the functional networks during the resting-state and continuous task execution. ${ }^{26-28}$ Particularly, naturalistic task paradigms, such as moviewatching ${ }^{29,30}$ and comprehension of a narrative story, ${ }^{3,4}$, 31 are interesting because of their ecological validity. Some studies have shown that FC is much reliable to demonstrate distinct individual differences when subjects involved in the naturalistic paradigm. For example, Londei et al. found that dynamics of the connectivity patterns within and toward somatosensory and motor areas are modulated by the degree of reproducibility of auditory speech material. ${ }^{32}$ A systematic reconfiguration of the cortical interactions, with changes in functional network assignments, has been demonstrated during challenging listening situations. ${ }^{33}$ In addition, Broderick and colleagues applied an approach based on a computational model to low-frequency noninvasively electroencephalographic (EEG) data recorded from subjects when they listened to narrative speech; and a prominent component was produced, which was very sensitive to whether or not subjects understood the speech they heard. Their results showed that the human brain responds to the contextual semantic content when successfully comprehending naturalistic speech. ${ }^{34}$ However, electrophysiological network connectivity between different brain regions for such low-frequency oscillations has been lacking for continuous speech. Furthermore, the condition-specific changes and increased reliability of functional brain connectivity may be induced by the task-dependent involvement of specific brain areas ${ }^{29,35}$ and reconfiguration of brain network may emerge during successful comprehension of narrative speech. $^{33}$ Based on these studies, we describe an approach for examining the brain network connectivity at low-frequency oscillations during speech comprehension. We hypothesized that distinct modes of brain networks would emerge and the reconfiguration of FC during comprehension of speech could be quantified in terms of systematic fluctuations in FC patterns.

In the present study, we used correlation of Hilbert envelope as a means to quantify FC between spatially separate brain areas. This metric has been used widely in recent years ${ }^{36-38}$ and has been characterized as an 'intrinsic mode' of functional coupling in the human brain. The high-density EEG were recorded and able to measure high spatiotemporal resolution networks. ${ }^{39}, 40$ We calculated the whole-brain connectivity between separate brain regions, which are predefined based on Desikan-Killiany atlas. ${ }^{41}$ To examine the reconfiguration of FC, we analyzed the fluctuations in grand averaged (over time) and dynamic (time-resolved) FC during listening to narrative speech and time-reversed speech (TR-speech). Here, the TR-speech can be used as a control to exclude brain processes induced by the lowlevel features of speech since it has the same long-term amplitude spectrum as normal speech but is not perceived as intelligible speech. ${ }^{42}$ Principal component analysis (PCA) was used to characterize the variations in FC patterns over subjects. PCA and related techniques have been applied to describe FC fluctuations during the resting-state, ${ }^{43}$ movie-watching ${ }^{29}$ and whole-brain connectivity dynamics. ${ }^{44,45}$ Based on the projections of scores on the principal components (PCs) for the individual subject, we identified FC modes dependent on the successful comprehension of speech condition. Furthermore, to examine whether fluctuations in grand averaged FC reflected a constant (temporally stationary) functional state or the occurrence of functional patterns altering over time, we extended our analysis beyond grand-average FC states and investigated the temporal fluctuations in FC states using dynamic FC based on a sliding-window technique. To examine the role of timelocked events on dynamic FC during speechcomprehension condition (similar to inter-subject synchronization), we estimated the similarity between instantaneous dynamic FC (each windowed FC) across conditions and runs. 


\section{Material and methods}

\subsection{Study design}

The open access EEG data used in this paper have been described in details elsewhere. ${ }^{34,} 42$ There were 10 subjects and they took part in two experiments. During the experiments, subjects were introduced to listen to a narrative speech and time-reversed speech, separately. The EEG data were recorded during listening task. In the first experiment (condition), subjects underwent 20 runs (trials), each of which was of the same length (less than 180 seconds), in which they listened to a professional audio-book edition of a classic American work of fiction (in this study we just used 2 runs to examine the dynamic organization during natural speech-comprehension). The audio was read by a single American English speaker. The runs retained the storylines, with neither duplicates nor discontinuities. During the audio-playing, the mean speech rate was 210 words/min. In a similar way, during the second experiment subjects were presented with the same runs in the same order, but with each of the speech stimuli played in reverse (time-reversed speech). All speech stimuli were played monophonically at a sampling rate of $44.1 \mathrm{kHz}$ using Sennheiser headphones in a sound-attenuated room when participants maintained fixation on a cross centered on a screen and minimized eye blinking and other movement activities.

\subsection{Data description and preprocessing}

During all experiments, dense array 128-channel EEG data (plus two mastoid channels) were recorded at a sampling rate of $512 \mathrm{~Hz}$ using a BioSemi ActiveTwo system. Offline, the data were filtered with a band-pass filter between 1 and $30 \mathrm{~Hz}$ since initial investigation of the frequency range uncovered that no useful information was observed in higher frequencies, and down-sampled to $128 \mathrm{~Hz}$. We then re-referenced EEG data to the common average channels ${ }^{46}$ in MATLAB2016b. To identify bad channels with artifacts noise, the EEG signals were visually inspected and the standard deviation of each channel time series was compared with that of the spherical surrounding channels. Bad channels with excessive noise were interpolated using a spherical spline model in EEGLAB. ${ }^{47}$ Independent component analysis (ICA) was performed to remove ocular EEG artifacts. $^{48,49}$

\subsection{Source reconstruction}

Following preprocessing, source localization was performed using an open access software Brainstorm. ${ }^{50}$ The forward model, describing the signal mode produced by the unit dipole at each predefined position on the brain model surface, was computed using the symmetric BEM method $^{51}$ based on default Colin27 MRI template provided by the Montreal Neurological Institute (MNI). Preprocessed data were adopted to compute the inverse model, which was estimated by the weighted Minimumnorm Estimation (wMNE). ${ }^{52}$ It has been proved that wMNE is well-established to estimate large-scale FC networks since it solves the volume conduction problems and thus reduces the correlation of spurious signal. ${ }^{53,54}$ When calculating the inverse operator, we adopted the configuration of parameters described in the previous study ${ }^{53}$ : (1) the current source orientations were constrained to perpendicular to the cortical surface; (2) the depth weighting algorithm was adopted to compensate for any deviations affecting the computation of superficial sources ${ }^{53,55}$; and (3) a regularization parameter, $\lambda^{2}=0.1$ was adopted to minimize numerical instability, reduce the wMNE sensitivity to noise, and effectively achieve a spatially smoothing estimation. ${ }^{53}$ In this procedure, source-level time series at 15,002 voxels were obtained. The cortical surface was then parcellated into 68 anatomical regions of interest (ROIs) based on the Desikan-Killiany atlas ${ }^{56}$ and the center of mass of each area was defined as a representative time series to be used to calculate FC.

\subsection{Functional connectivity estimation}

We aimed to perform an all-to-all whole-brain FC analysis by estimating connectivity between all possible pairs of Desikan-Killiany regions. In M/EEG, a significant confound of source level connectivity is that the ill-posed inverse problem plus inaccuracies in the forward solution, leads to a degree of spatial blurring and mislocalization of sources. ${ }^{57,58}$ This confound means that two source-reconstructed time series (e.g. from two brain regions) may be significantly correlated, purely due to 'signal leakage' (for review see Ref. ${ }^{38}$ ). The estimated connectivity between separate brain regions may not be accurate without careful control. ${ }^{58}$ Signal leakage issue has been well investigated and there are now a lot of techniques for leakage reduction. ${ }^{37,57,59}$ Most approaches are based on the fact that leakage manifests as a zero-time lag linear superposition of underlying signals so that 
although the true zero-lag connection is sacrificed, orthogonalization of source-reconstructed signals can effectively remove leakage. ${ }^{58}$ Colclough et al. recently proposed an effective method of simultaneously orthogonalizing over a set of multiple brain regions. ${ }^{60}$ Based on their study, here, signals (time-courses) from all $\mathrm{N}=68$ brain areas are symmetrically orthogonalized in a single calculation. The complete mathematical details of the procedure can be found in previous study. ${ }^{60}$ In brief, two steps need to be conducted in the method: First, find a set of orthogonal time-courses that are closest to the data matrix and have a simple analytical solution. Second, the solution is finessed by iteratively adjusting the lengths and orientations of the corrected vectors until the solution is as close as possible to the uncorrected time-courses. ${ }^{61}$ This results in a set of matrices, whose rows consist of the orthogonalized time-courses for all 68 Desikan-Killiany brain areas. Following signal leakage correction, the Hilbert transformation was applied to extract the amplitude envelopes of the time-courses. The FC matrices were constructed based on Pearson correlation coefficient between all pairs of the amplitude envelopes of ROIs in terms of grand average FC. To extract dynamic FC (time-resolved FC), we applied a sliding window approach. ${ }^{44,62}$ The window length was set as $5 \mathrm{~s}$ and the overlap was $4 \mathrm{~s}$ between two adjacent windows. Within each window, we calculated connectivity between all pairs of Desikan-Killiany regions. It should be noted that the signal leakage reduction step was performed on each time window separately (separate orthogonalization for each window), rather than on the whole time series during grand average FC analysis. This conduction can be explained in previous study, ${ }^{63}$ where it has proved that leakage relies heavily on signal to noise ratio (SNR) and the SNR is different in different time windows.

\subsection{Principal component analysis}

During grand averaged FC analysis, the FC matrices were calculated based on Pearson correlation between all pairs of the amplitude envelopes of 68 ROIs (over whole time) for all subjects and runs (see Section Functional connectivity estimation).

To perform PCA, the lower triangular parts of FC (i.e. $68 \times(68-1) / 2$ connections $)$ matrices were concatenated across subjects/runs $(10 \times 4$ subjects/runs $)$ resulting in the group-level connectivity matrix with dimensions $2278 \times 40$ (number of connections $\times$ number of subjects/runs). PCA was then performed to the resulting group-level connectivity matrix. To determine the components which reflect only noise, the PCA analyses were repeated for 1000 surrogate time-courses for each subject/run. The surrogate time-courses of each individual subject were phase-randomized and the properties of the surrogate time-courses were preserved in spectral domain. ${ }^{29,} 64$ The dimensionality of the data was represented by the proportion of explained variance of the principal components (PCs) that are greater than those of the surrogates. Since the data were decomposed by PCA into orthogonal axes with related projections (scores) of each individual observation (subject or run in this study), we used these projections scores to characterize the principal components. The PC associated with speech-comprehension condition was considered as the one demonstrating clear separation between different conditions and significant difference levels based on the PC projection scores (i.e., the scores higher than 0 represented the speech-comprehension runs, whereas the scores less than 0 presented the timereversed speech runs).

To examine the consistency of PCs, the analysis was repeated using 2 separate runs. For both runs, the grouplevel connectivity matrices contained the concatenated lower triangular FC matrices of 1 time-reversed speech run and 1 speech-comprehension run (i.e., $2278 \times 20$ matrices). The consistency was characterized as Pearson Correlation of the components and their projection scores across runs (See Figure 1G-H). 

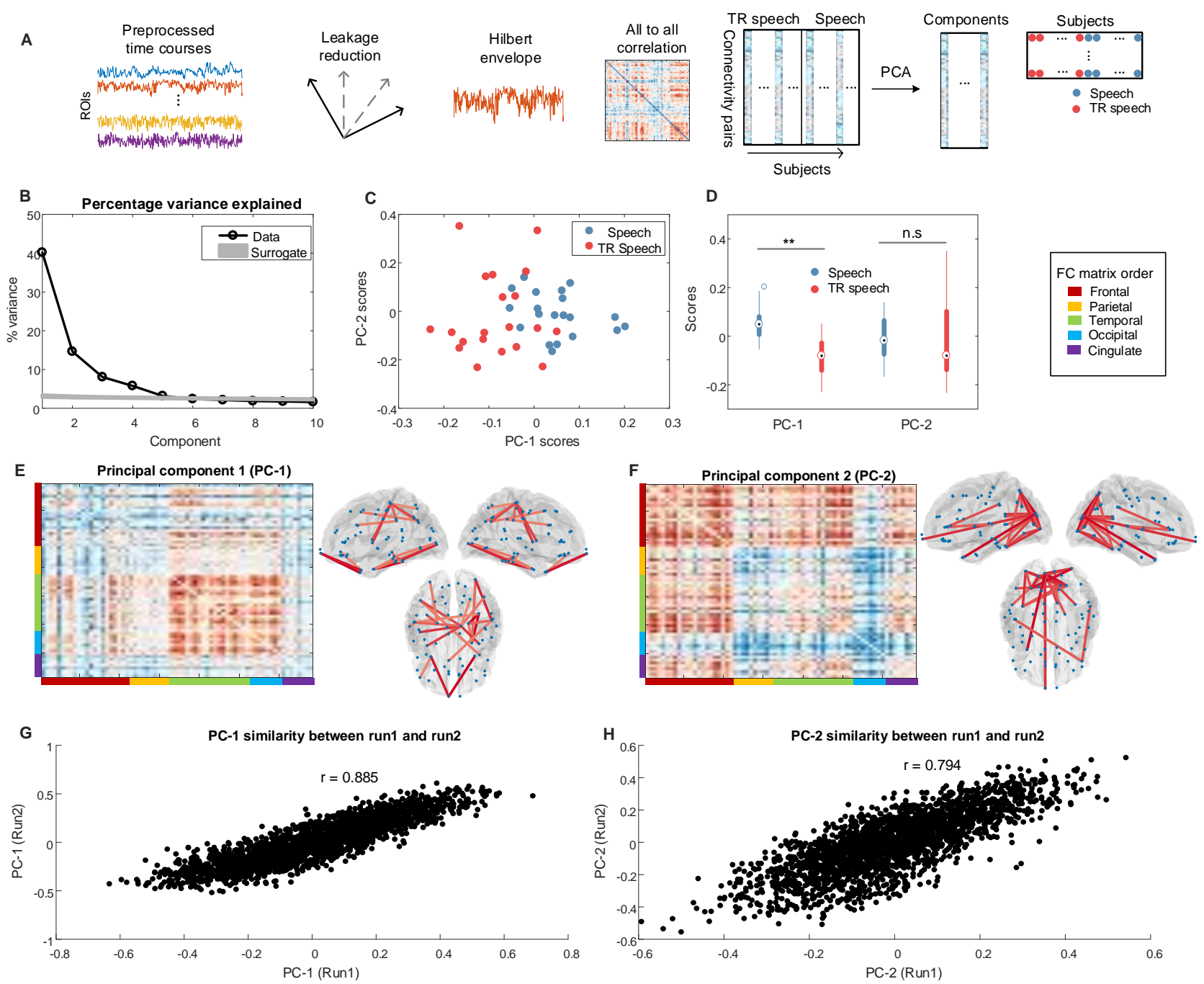

Fig. 1. A The analysis pipeline using principal component analysis (PCA). The time-courses of ROIs were firstly corrected to remove signal leakage; then, the FC matrix was constructed using Hilbert envelope correlation; finally, the PCA was performed over FCs of 2 time-reversed speech and 2 speech-comprehension condition concatenated across 10 subjects. B Explained variance by each PC (black line) and phase-random surrogates (gray line). The first 4 PCs were above the noise level (Surrogate data); the first PC (E) explained $39.8 \%$ of the variation, while the second (F) explained 14.6\%. C The projections of first two PCs and D Boxplot of first 2 PCs projections, showing that the first component is dependent to speech-comprehension condition. E-F The first 2 components and their 3-D representations with threshold (top 5\%) for visualization. G-H The similarity of the first 2 PC between two separated run analysis.

\subsection{PCA trajectories of dynamic FC}

The PCA was repeated for average dynamic FC to establish the link between grand averaged FC (whole time correlation) and dynamic FC. After determining the grand average condition-specific dynamic FC component, we applied PCA to concatenated dynamic FC matrices over time for each subject (i.e., 2 timereversed speech and 2 speech-comprehension runs). The condition-specific temporal components (connectivity) were determined as the PC exhibiting the highest similarity to the grand average condition-specific dynamic FC components. Then, we measured the trajectories (i.e., fluctuations of PC scores over time) of the condition-specific temporal components of individuals. In this study, the term "trajectory" was better than "scores" to highlight the fact that the PCA was applied to time-concatenated connectivity matrices. We examined whether the PC exhibiting highest similarity to 
the condition-specific connectivity differentiated between time-reversed speech and speechcomprehension trajectories. We characterized the condition-specific differences by computing the average Euclidean distance between the median trajectories of the time-reversed speech and speech-comprehension conditions. The Euclidean distances between median trajectories were defined as the squared difference between median PC projection scores of time-reversed speech and speech-comprehension trajectories. For each subject, to evaluate the significance of the distinction, we compared the condition-specific Euclidean distance with those of the surrogates. We randomly shuffled the trajectories of each individual subject and then reassigned them into two groups. The $\mathrm{p}$-values were assessed by comparing the distance between conditionspecific trajectories with the distance of the surrogates. Since the trajectories of each individual PC are timedependent, we evaluated the distinction between different conditions across subjects by computing the median distances across runs and conditions. For each individual subject, the median distance of trajectory between time-reversed speech and speechcomprehension conditions was computed. Next, the distances between two separate runs of time-reversed speech and speech-comprehension conditions were computed. Finally, we adopted permutation tests to compare the average distance across runs and conditions.

\subsection{Dynamic FC similarity across conditions and runs}

During continuous perception, human brain automatically divides experiences into discrete events. ${ }^{65}$ To examine the role of time-locked events on dynamic FC in speech-comprehension condition (similar to intersubject synchronization), we estimated the Pearson similarity between instantaneous dynamic FC (each windowed FC) across conditions and runs. For each time window, we computed the Pearson similarity between the FC matrix of a single subject $(\mathrm{k})$ and the average FC across the remaining of the subjects $(\mathrm{n} \neq \mathrm{k})$. The average dynamic $\mathrm{FC}$ was computed to test the $\mathrm{FC}$ similarity in 3 different circumstances: across conditions (i.e., if subject $\mathrm{k}$ is at time-reversed speech run 1, the average dynamic FC was computed for speech-comprehension run 1), across runs (i.e., if subject $\mathrm{k}$ is at time-reversed speech run 1, the average dynamic FC was computed for timereversed speech run 2) and within runs (i.e., if subject $\mathrm{k}$ is at time-reversed speech run 1, the average dynamic FC was computed for time-reversed speech run 1) (Fig 3A).

\subsection{Statistical analysis}

The comparisons between conditions (time-reversed speech vs. speech-comprehension) were performed using permutation tests since the size of samples remines relatively small. During the permutation tests, the randomization was also carefully controlled to keep the dependence across two conditions.

To evaluate the association between measures, Spearman's correlations were used due to limited number of samples. Pearson correlation was applied as a measure of similarity between connectivity matrices. (i.e., PC scores, FCs, and dynamic FCs).

\section{Results}

To characterize the fluctuations in FC across subjects during time-reversed speech and speech-comprehension conditions, PCA was applied over subjects (Figure 1A). PCA decomposed high-dimensional group-level connectivity matrices into orthogonal principal components that explained the most variance of the data. The projections provided a score for each individual observation (i.e., subject/run) along the PCs. We performed PCA on concatenated vectorized connectivity matrices for all subjects during two separate runs of timereversed speech and speech-comprehension conditions. We then examined the scores (i.e., projections of PCs by individual subjects) during two conditions.

\subsection{Distinct modes of variation in FC during speech comprehension}

The first principal component (PC-1), explaining 39.8\% of the variance (Fig. 1B), was able to distinguish the speech-comprehension condition from time-reversed speech condition. The projection scores of PC-1 were significantly different between two conditions ( $\mathrm{p}<$ 0.001) (Fig. 1C-D). We considered this principal component as a condition-specific PC. This result demonstrated that the condition-specific changes in FC can be explained with a single pattern of variation (PC1). The second principal component (PC-2) (Fig .1B), explaining $14.6 \%$ of the variance, reflected a FC mode that was preserved across runs. There was no significant difference in the scores of PC-1 between two conditions (Fig. 1C-D). This result showed that the principal 
A

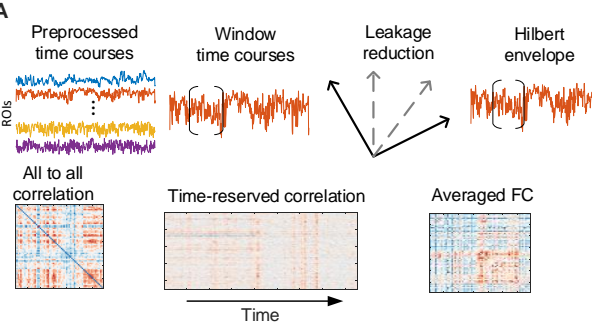

B Average FC component
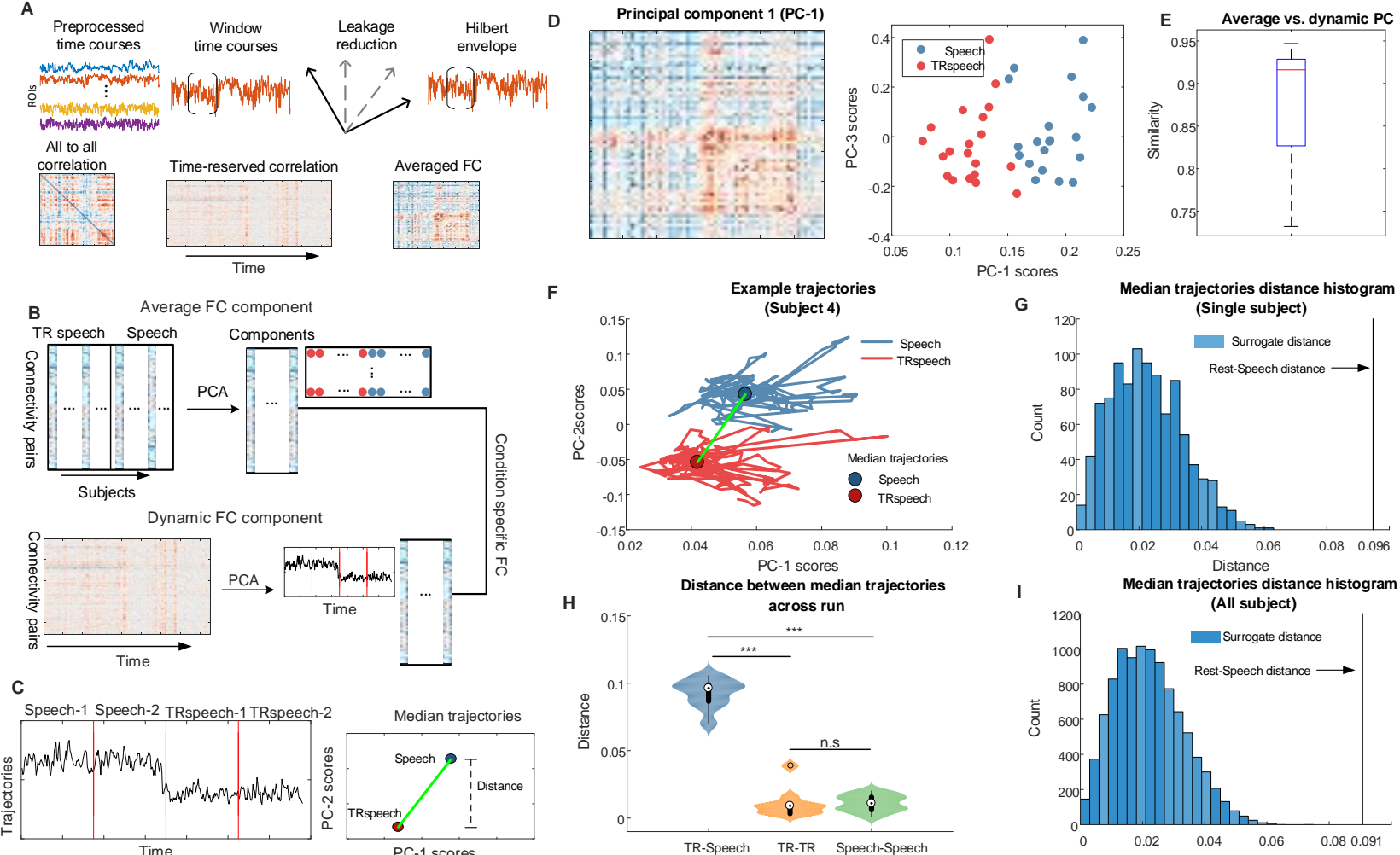
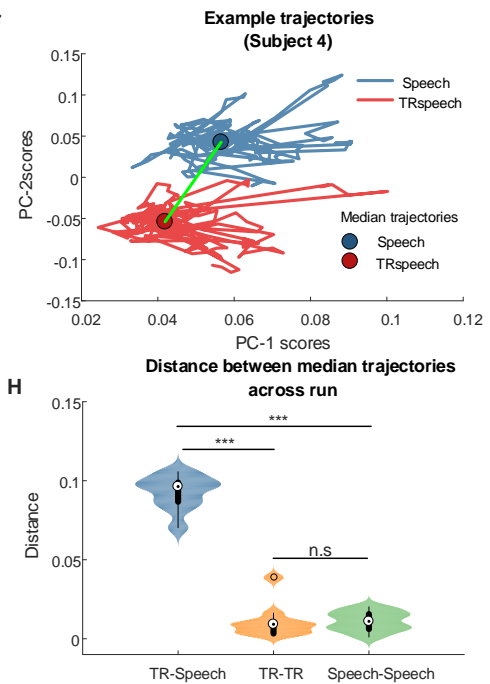
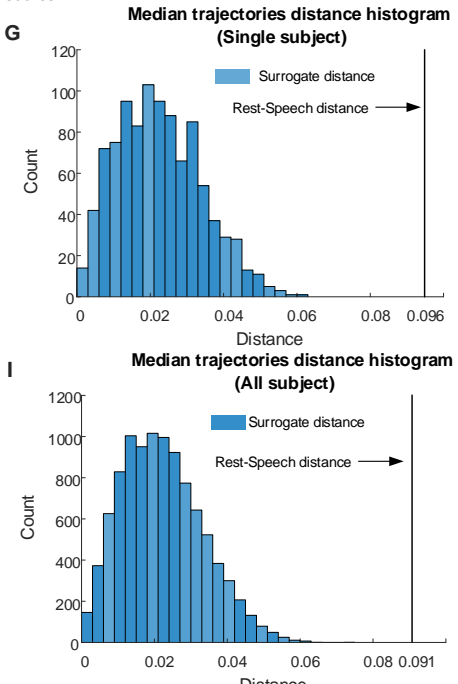

Fig. 2. FC based on a sliding-window approach. A The pipeline of dynamic FC analysis. The source-reconstructed time-courses were leakage-corrected within each window (window length $5 \mathrm{~s}$, overlap $4 \mathrm{~s}$ ). Dynamic FCs were estimated based on windowed Hilbert envelop correlation. B The pipeline describing the PCA applied to average dynamic FCs across subjects (top), where the static adjacent matrixes were concatenated across subjects, and dynamics FCs across time for each subject (bottom). A condition-specific component was determined based on the maximum similarity between dynamic FC components and average condition-specific FC component (D). The grand average component and dynamic condition-specific component were very similar across subjects (E). C Based on the trajectories of condition-specific dynamic FC components, the distance between the median trajectories of time-reversed speech (TR speech) and speech-comprehension (Speech) conditions were computed. F Example trajectory for single individual (Subject 4). G The distance between the median trajectories of TR speech and Speech conditions compared to the histogram of the distances for 1000 randomly split trajectories (Subject4) and $\mathbf{I}$ for all subjects. H The median trajectory distances between TR speech and Speech conditions, between 2 TR speech runs and between 2 Speech runs. The distance between conditions was significantly higher than the distance between runs (permutation tests, 10000 permutations). $* * *$ represents $\mathrm{p}<0.0001$, n.s. represents $\mathrm{p}>0.05$.

component of variation in $\mathrm{FC}$ reflected the common connectivity pattern over two conditions.

PCA analysis was repeated for 1000 surrogate FC matrices across all subjects to determine the components explaining a significant fraction of variance (see section Principal component analysis). The explained variance of the first 4 components was larger than the explained variance of surrogate FC matrices. Considering a large amount of variance explained by the first 2 PCs, we chose the 2 PCs for further analysis. The rest of the components did not exhibit any specificity regarding the speechcomprehension condition and were not presented.
To examine the consistency of the condition-specific PCs over runs, PCA analysis was repeated for each run separately and the Pearson similarities between PCs across runs were quantified. For each run, the conditionspecific PCs showing high consistency across runs were identified. The similarities between PCs were significantly correlated across runs for condition-specific $\mathrm{FC}$ and common $\mathrm{FC}$ ( $\mathrm{r}=0.885$ for $\mathrm{PC}-1$ component, $\mathrm{r}=0.794$ for PC-2 component) (Fig. 1G-H). These results showed that the condition-specific PC and the related projection scores were consistent across runs. 


\subsection{Condition-specific FC trajectories in dynamic FC}

The grand average FC metric is not able to distinguish between a temporally stationary pattern of FC and fluctuations in FC. Thus, we analyzed dynamic fluctuations in FC (also known as time-resolved FC). In the current study, we examined the hypothesis that brain FC continuously reconfigured during speechcomprehension. The dynamic FC was estimated based on a sliding window technique. We used envelope correlation as a means to quantify connectivity between spatially separate brain regions (see section Functional connectivity estimation). This metric has been used extensively in recent years and has been described as an 'intrinsic mode' of functional coupling in the human brain. Here, we set window length as $5 \mathrm{~s}$ and overlapped with $4 \mathrm{~s}$ between adjacent frames (see section Discussion about the window length). First, the source-localized time series of separate brain regions were segmented into overlapping time windows. Second, leakage reduction step was applied to each window, separately. Hilbert envelope was extracted from the corrected time series. Finally, we computed 'all-to-all' connectivity between separate brain regions (Fig. 2A).

To establish the link between the dynamic FC analyses and grand average FC (whole time correlation). The average dynamic FC across time was calculated and PCA was performed over subjects. The analysis results suggested that the PC based on averaged dynamic FC also showed condition specificity (Fig. 2). In addition, condition-specific PC of average dynamic FC was similar to those grand average FCs ( $\mathrm{r}=0.92$ ) (Fig. 2E). Thus, the condition specific FC patterns from average dynamic connectivity were in line with those based on grand average FC.

For each participant, PCA was applied to the dynamic FCs over time (Fig. 2B). We considered the conditionspecific component for each individual subject as the one that was the highest correlated with the grand average condition-specific component (Fig. 2D). For most subjects, the trajectories (PC projection scores) of the condition-specific components reflected a significant difference between conditions (Fig. 2H). For each subject, we characterized the condition-specificity by comparing the median of trajectories (median PC projection scores) during the time-reversed speech and the speech-comprehension conditions (Fig. 2C). Then, we measured the Euclidean distance between the median trajectories of TR-speech and speech conditions (Fig. 2C). We compared the distance between TR-speech and speech median trajectories with the distance between 1000 trajectories of randomly shuffled groups (Fig. 2GI). The results of all subjects demonstrated a significantly larger distance between TR-speech and speech trajectories than any other trajectories of randomly shuffled groups $(\mathrm{p}<0.0001)$ (Fig. 2I). Due to the timedependence of the condition-specific PC trajectories, we evaluated the significance of the median distances of trajectory between conditions/runs across subjects. We observed that the median distance across conditions (i.e., TR-speech/speech conditions) was significantly greater than the median distance across runs (i.e. TR-speechrun1/TR-speech-run2 and speech-run1/speech-run2) ( $\mathrm{p}<0.0001$, permutation tests, 10000 permutation runs) (Fig. 2H). We observed no significant difference between the median distance across runs for time-reversed speech and speech-comprehension conditions (Fig. 2H). These results suggested the emergence of a preserved FC mode during speech-comprehension condition at a short time scale.

\subsection{Condition-specific FC patterns within and across runs}

The first principal component (PC-1), explaining 39.8\% of the variance (Fig. 1B), was able to distinguish the speech-comprehension condition from time-reversed speech condition. The projection scores of PC-1 were significantly different between two conditions ( $\mathrm{p}<$ 0.001) (Fig. 1C-D). To investigate the role of timelocked events on FC dynamics during speechcomprehension (similar to inter-subject synchronization analysis), we computed the Pearson similarity between dynamic FCs over conditions and runs. Briefly, for each time window, we calculated the similarity between the FC matrix of an individual subject $(\mathrm{k})$ and the average FCs across the remaining subjects $(n \neq k)$. The average FCs were computed to examine the FC matrix similarity in 3 different cases: across conditions (e.g. if subject $\mathrm{k}$ is at time-reversed speech run 1, the average FC matrix was computed for speech-comprehension run 1), across runs (e.g., if subject $\mathrm{k}$ is at time-reversed speech run 1 , the average FC matrix was computed for time-reversed speech run 2) and within runs (e.g., if subject $\mathrm{k}$ is at timereversed speech run 1 , the average FC matrix was computed for time-reversed speech run 1) (Fig. 3A). 
A

Run: $\{1,2\}$

Condition:\{Speech, TRspeech\}

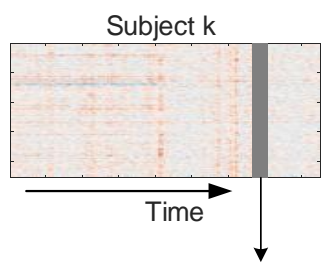

$R(t)$

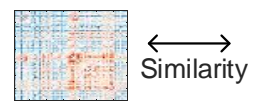

Different condition

Remaining subjects $(n \neq k)$

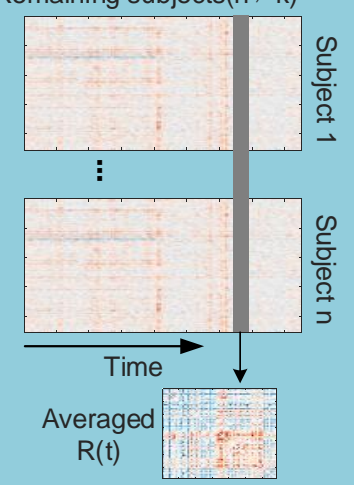

-Across condition
Same condition

Different run

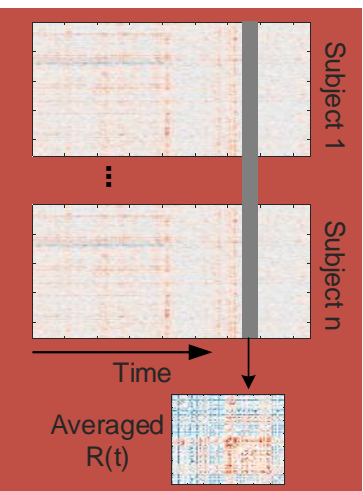

Same condition

Same run

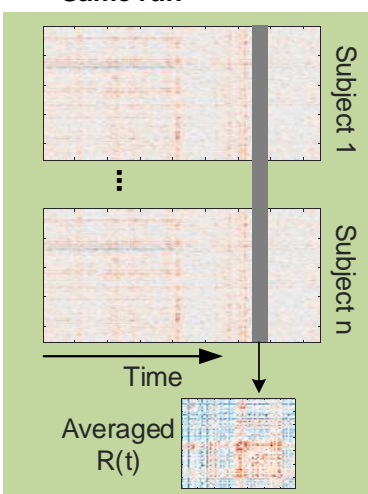

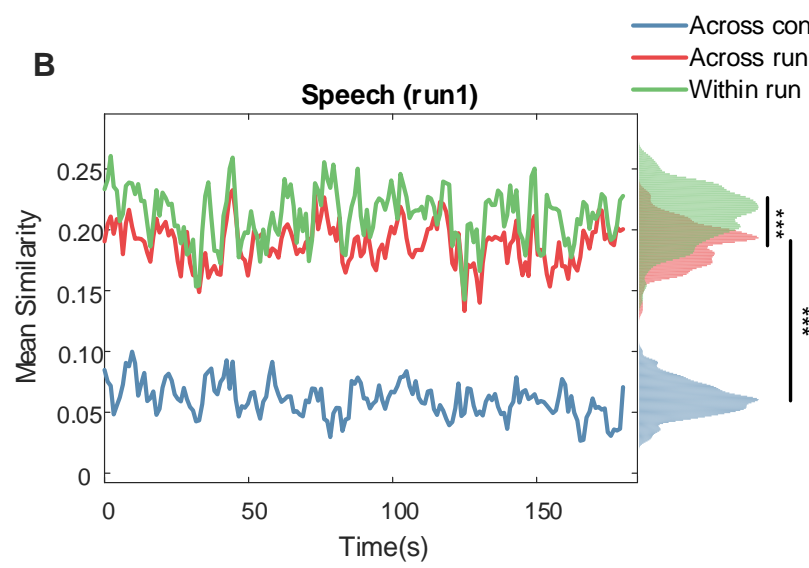

D

TRSpeech (run1)

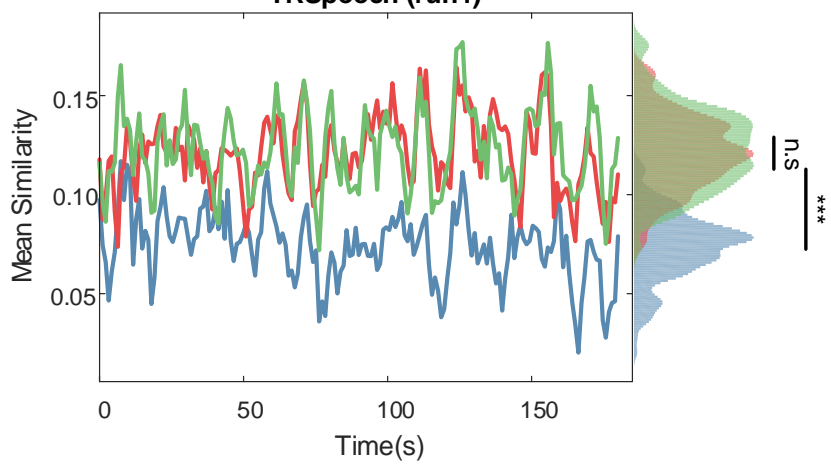

E TRspeech (run2)
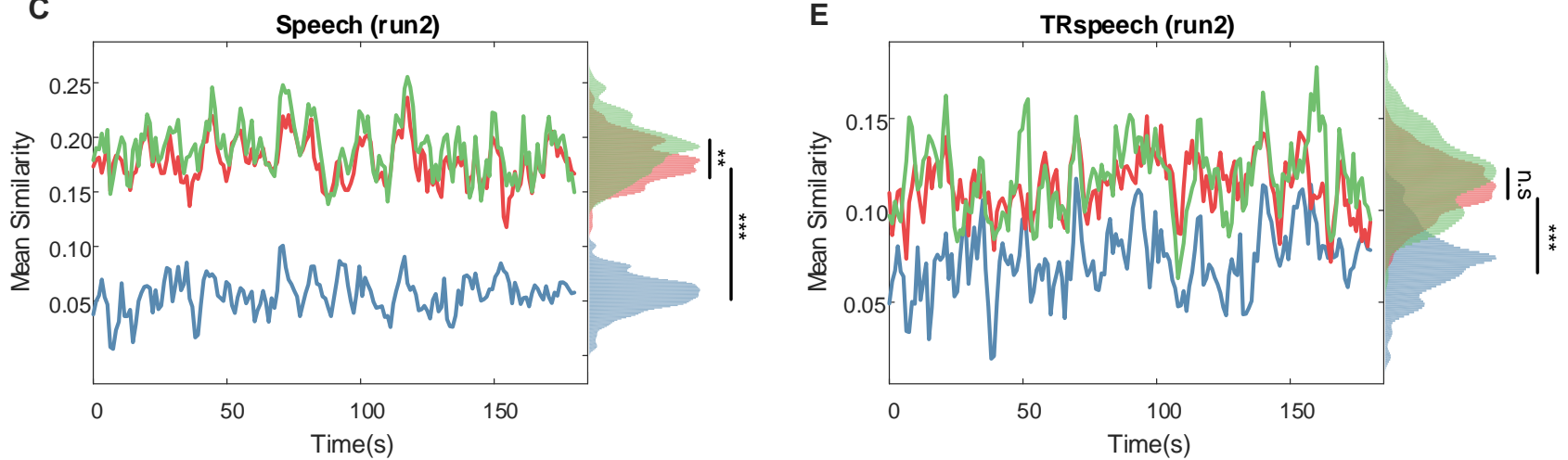

Fig. 3. Time-resolved similarity between dynamic FCs across conditions and runs. A The analysis pipeline. For each individual subject the dynamic FC at each window was compared to the average dynamic FC across the rest of subjects at the same window. Blue lines/shades represent that the average dynamic FCs were computed for different condition (i.e. if subject $\mathrm{k}$ is TR speech, average dynamic FC were computed across subjects for Speech condition (excluding subject $\mathrm{k}$ )). Red lines/shades represent that the average dynamic FCs were computed for the same condition but different runs (i.e. if subject $\mathrm{k}$ is at TR Speech in run 1, average dynamic FC were computed for the TR speech run 2). Green lines/shades represent that the average dynamic FCs were computed for the same condition and the same run (i.e. if subject $\mathrm{k}$ is at TR speech in run 1, average PLVs were computed for the TR speech run 1). B-C During speech-comprehension, the similarity between dynamic FCs was significantly lower across conditions, but the similarity between FCs was significantly higher within runs compared to across runs. D-E During TR speech the similarity between dynamic FCs were significantly lower across conditions, but there was no significant difference between the similarities across runs. The histograms indicate the distributions of similarity measures over time, whereas *** represents the $\mathrm{p}<0.0001$, ** represents the $\mathrm{p}<0.001$ assessed by permutation tests across subjects. n.s. represents $p>0.05$. 
The similarity across runs both for TR-speech and speech-comprehension conditions was significantly larger than the similarity across conditions $(p<0.001$ for both runs: permutation tests, 10000 permutation runs) (Fig. 3). This result confirmed the continuous functional reconfiguration during speech-comprehension condition. Additionally, this result also demonstrated that during speech-comprehension, the similarity between dynamic FC was higher, even when the subjects were listening to different semantic content. The average similarity between dynamic FCs demonstrated no significant difference across runs for resting-state runs $(\mathrm{p}=0.54$ for run $1, \mathrm{p}=0.28$ for run 2 , permutation tests, 10000 permutations) (Fig. 3D-E). In contrast, the average similarity between dynamic FCs was significantly higher for the same speech run than across runs $(\mathrm{p}<0.0001$ for run $1, \mathrm{p}<0.001$ for run 2, permutation tests, 10000 permutations) (Fig. 3B-C). These results showed that the dynamics of FC during speech-comprehension reflected both the effects of time-locked events and a continuous reorganization of brain networks.

\section{Discussion}

This paper has investigated the reconfiguration of functional connectivity (FC) during speechcomprehension condition. The results demonstrated that FC dynamic pattern under speech-comprehension varies along with a single mode of variation. During the comprehension of natural speech, the connectivity pattern captures the variations over subjects, which emerges as a continuous brain functional state across time.

We adopted PCA to characterize the variations in FC across subjects and conditions. We found that one of the PCs (PC-2) reflected the common pattern of variations in both conditions, whereas another PC (PC-1) reflected the difference between time-reversed speech and speechcomprehension conditions. The patterns of the components characterized the intra- and interhemispheric connectivity within occipital and temporal regions as well as their connections with frontal and parietal regions, which are line with increased brain connectivity in language/auditory networks. ${ }^{66,67}$ These results demonstrated that the increased communication between separate brain regions associated with auditory processing and attention are primarily driven by the timelocked events during comprehension of natural speech.
This characterization is consistent with the large-scale auditory and sensory-motor networks emerging during comprehension of natural, narrative speech. ${ }^{32}$ The condition-specific component (PC-1) demonstrated increased connectivity within sensory-motor areas and reduced connectivity between frontal-parietal brain regions and cingulate. The enhanced sensory-motor areas connectivity reflects the ability to transform auditory speech into appropriate sensory and motor representations. These results are in line with previous studies showing brain functional reorganization during comprehension of speech. ${ }^{32,} 33$ Furthermore, the enhanced frontal-temporal connectivity may indicate a strong functional cross-talk between ventral attention and auditory regions. Previous studies also observed the reconfiguration of a frontal-temporal network in adaptation to cued speech comprehension, where related frontal-temporal cortical regions were referred to as the auditory-control network. ${ }^{33}$

The result about emerging of a condition-specific pattern in grand averaged FC may be not sufficient to draw a conclusion about the reorganization of FC during speech comprehension. Therefore, we examined how the condition-specific component relates to the dynamic FC. We applied a sliding window approach combining with Hilbert envelop correlation between brain regions to characterize dynamic FC over time. The findings also showed condition-specific $\mathrm{PC}$ on grand average dynamic FC across subjects and individual dynamic FC over time. The trajectories of the condition-specific PCs demonstrated that this PC might emerge as a stationary pattern during comprehension of speech. This conclusion was resulted from analyzing the similarity between instantaneous dynamic FC and the average dynamic FC across individuals under different runs/conditions. The similarity was significantly higher when the individuals were involved in the same conditions (i.e., TRspeech/TR-speech and speech/speech) than they were in different conditions (i.e. TR-speech/speech). In addition, only during speech comprehension, the similarity of the dynamic FC was higher for individuals in the same run (i.e. run 1/run 2) than in the different runs (i.e. run 1/run 2). Overall, these results demonstrated that whole-brain connectivity is reorganized over time. Previous studies also observed that dynamics of FC states are highly stable relying on the narrative of a story although the connectivity patterns were similar over time. ${ }^{4}$ Our results showed that the dynamics of the distinct connectivity 
states might display time-locked events. Previous studies also demonstrated that humans automatically segment experiences into discrete events during realistic, continuous perception. ${ }^{65} \mathrm{We}$ speculated that the reconfiguration of the brain networks in higher-order regions might reflect the adaptation of the brain's intrinsic networks to coordinate the large-scale flow of information during speech comprehension. The dynamics of the condition-specific component may suggest that these changes of the FC patterns are related to higher-level processing of the narrative.

The PCA analysis in our study revealed two different modes of fluctuations that were related to the FC condition-specific variations. Although the PC-2 was consistent with the variations in empirical and model data, the PC-1 demonstrated a substantial conditiondependence. Furthermore, the PC-1 exhibited a similar pattern with typical of default mode network (DMN), which involves the medial frontal, temporal, and cingulate cortices. These local regions continuously change their module property in adaptation to the comprehension of speech. ${ }^{33} \mathrm{We}$ speculated a group of temporal and cingulate cortices merge with other temporal regions from the default mode network and form a new common mode during comprehension of speech. This result is consistent with the robust and reproducible reconfiguration of default mode network during narrative comprehension. ${ }^{4}$

From the methodological considerations, although a lot of methods have been proposed to EEG analysis for various applications, such as detection of epilepsy, Alzheimer's disease and so on, ${ }^{68-71}$ the approach proposed in the current paper provided an analysis framework that used $\mathrm{M} / \mathrm{EEG}$ imaging technique to investigate the reorganization of brain connectivity during naturalistic paradigm (e.g. music-listening, movie-watching, story-listening and speech comprehension). During analysis for the dynamic FC, the window length (here $5 \mathrm{~s}$ ), one core parameter, requires setting. This parameter setting warrants further discussion. An appropriate selection of window length is important and stands for a trade-off between temporal resolution and the accuracy of the derived connectivity matrices. ${ }^{38,58}$ In this study, elements of the connectivity matrices are derived from the temporal correlation of envelope time-courses within the window. It is well known that the accuracy of the correlation between two signals (r) is associated with the number of degrees of freedom $(\eta)$. Specifically, if assuming no underlying correlation between two time series then the standard deviation of correlation, $\sigma(r)=1 / \sqrt{\eta}$. In other words, the noise in adjacency matrices is increased as $\eta$ is decreased. Furthermore, the number of degrees of freedom in a fixed-window time series is independent of the numbers of sample points. In the view of Fourier theory, an upper limit on degree of freedom for envelope data is given by $\eta=B_{w} \delta$, in which $\delta$ is the window length and $B_{w}$ denotes bandwidth of the carrier signal. Typically, bandwidth is set by the scientific question to be asked. For example, previous studies were interested in beta band networks for self-paced motor study. ${ }^{58,72}$ Here, $\sigma(r)=0.08$, which was deemed acceptable. Future studies should keep this computation in mind.

Finally, it should be noted that there are several limitations when interpreting the results in the current study. The most important limitation of the current study is the small number of the sample even though our results are validated using both surrogate data and permutation tests. Therefore, the results need replication in a separate independent dataset. Furthermore, the design of this study from previous studies did not allow us to compare the obtained results with other conditions (i.e. different narrative speech). Also, the current study did not allow us to compare the results with resting-state since the open access data did not include the resting-state condition. Future studies may study the variants of speechcomprehension condition, different task performance or other naturalistic paradigms (e.g., free music-listening, movie-watching, etc.). When analyzing large-scale neurophysiological networks using MEG/EEG, the common problem is non-physiological spread of electrical activity through volume conduction causing spurious correlations between signals. ${ }^{73}$ Here, we used signal leakage reduction technique to address this problem. This technique has been well studied and believed to be an optimal approach for large-scale functional connectivity analysis. ${ }^{38,58,60}$ Yet, it should be noted that the technique can only reduce the volume conduction problem, not address it completely. Another limitation of this study is the use of coarse (34 regions per hemisphere), anatomically defined parcellation based on default MNI MRI template. The simultaneous individual MRI scanning may facilitate the coregistration for EEG electrode. Additionally, we only examined the functional connectivity at the band of 1-30 $\mathrm{Hz}$ as it has been shown low-frequency oscillations 
contribute to the naturalistic speech comprehension. ${ }^{34,42}$ Recently it has been suggested that functional connectivity is dependent on frequency band during the task execution such as working-memory and self-paced motor task. ${ }^{61,} 74$ In a future study, we will examine whether functional connectivity during speech comprehension is frequency-dependent or not.

\section{Conclusion}

The brain FC is highly dynamic and able to adjust topology on a very fine time-scale during changing environment. Here, we proposed an analytical approach and investigated the reconfiguration of the brain functional networks during comprehension of natural, narrative speech. Our findings demonstrated that FC dynamic patterns under speech-comprehension vary along with a single mode of variation. Furthermore, our analysis method seems valuable for studying the reorganization of dynamic brain networks based on $\mathrm{M} / \mathrm{EEG}$ data during natural task experiments.

\section{Acknowledgements}

This work was supported by the National Natural Science Foundation of China (Grant No. 91748105\&81471742), the Fundamental Research Funds for the Central Universities [[DUT2019] in Dalian University of Technology in China, and the scholarship from China Scholarship Council (No. 201600090042; No. 201600090044).

\section{References}

1. Simony E., Honey C. J., Chen J., Lositsky O., Yeshurun Y., Wiesel A. and Hasson U. 2016, "Dynamic reconfiguration of the default mode network during narrative comprehension," Nature communications 7, 12141.

2. Liu C., Abu-Jamous B., Brattico E. and Nandi A. K. 2017, "Towards tunable consensus clustering for studying functional brain connectivity during affective processing," International journal of neural systems 27, 1650042.

3. Lerner Y., Honey C. J., Silbert L. J. and Hasson U. 2011, "Topographic mapping of a hierarchy of temporal receptive windows using a narrated story," J Neurosci 31, 2906-15.

4. Simony E., Honey C. J., Chen J., Lositsky O., Yeshurun Y., Wiesel A. and Hasson U. 2016, "Dynamic reconfiguration of the default mode network during narrative comprehension," Nat Commun 7, 12141.

5. Deco G., Tononi G., Boly M. and Kringelbach M. L. 2015, "Rethinking segregation and integration: contributions of whole-brain modelling," Nature Reviews Neuroscience 16, 430 .
6. Tononi G. 2004, "An information integration theory of consciousness," BMC neuroscience 5, 42.

7. Bassett D. S., Wymbs N. F., Porter M. A., Mucha P. J., Carlson J. M. and Grafton S. T. 2011, "Dynamic reconfiguration of human brain networks during learning," Proc Natl Acad Sci U S A 108, 7641-6.

8. Schmidt C., Piper D., Pester B., Mierau A. and Witte H. 2018, "Tracking the reorganization of module structure in time-varying weighted brain functional connectivity networks," International journal of neural systems $\mathbf{2 8}$, 1750051

9. Alarcón G., Jiménez-Jiménez D., Valentín A. and MartínLópez D. 2018, "Characterizing EEG Cortical Dynamics and Connectivity with Responses to Single Pulse Electrical Stimulation (SPES)," International journal of neural systems 28, 1750057.

10. Ahmadlou M., Adeli A., Bajo R. and Adeli H. 2014, "Complexity of functional connectivity networks in mild cognitive impairment subjects during a working memory task," Clinical Neurophysiology 125, 694-702.

11. Yuvaraj R., Murugappan M., Acharya U. R., Adeli H., Ibrahim N. M. and Mesquita E. 2016, "Brain functional connectivity patterns for emotional state classification in Parkinson's disease patients without dementia," Behavioural brain research 298, 248-260.

12. Ahmadlou M. and Adeli H. 2011, "Functional community analysis of brain: A new approach for EEG-based investigation of the brain pathology," Neuroimage 58, 401408.

13. delEtoile J. and Adeli H. 2017, "Graph theory and brain connectivity in Alzheimer's disease," The Neuroscientist 23, 616-626.

14. Biswal B., Zerrin Yetkin F., Haughton V. M. and Hyde J. S. 1995, "Functional connectivity in the motor cortex of resting human brain using echo - planar MRI," Magnetic resonance in medicine $\mathbf{3 4}, 537-541$.

15. Brookes M. J., Wood J. R., Stevenson C. M., Zumer J. M., White T. P., Liddle P. F. and Morris P. G. 2011, "Changes in brain network activity during working memory tasks: a magnetoencephalography study," Neuroimage 55, 1804 15.

16. Li F., Peng W., Jiang Y., Song L., Liao Y., Yi C., Zhang L., Si Y., Zhang T. and Wang F. 2019, "The dynamic brain networks of motor imagery: time-varying causality analysis of scalp EEG," International journal of neural systems 29, 1850016

17. Correas A., Rodríguez Holguín S., Cuesta P., LópezCaneda E., García-Moreno L. M., Cadaveira F. and Maestú F. 2015, "Exploratory analysis of power spectrum and functional connectivity during resting state in young binge drinkers: A MEG study," International journal of neural systems 25, 1550008.

18. Ahmadlou M., Adeli H. and Adeli A. 2012, "Graph theoretical analysis of organization of functional brain networks in ADHD," Clinical EEG and neuroscience 43, 5-13.

19. Smith S. 2016, "Linking cognition to brain connectivity," Nat Neurosci 19, 7-9.

20. Smith S. M., Nichols T. E., Vidaurre D., Winkler A. M., Behrens T. E., Glasser M. F., Ugurbil K., Barch D. M., Van Essen D. C. and Miller K. L. 2015, "A positive-negative 
mode of population covariation links brain connectivity, demographics and behavior," Nat Neurosci 18, 1565-7.

21. Ahmadlou M. and Adeli H. 2017, "Complexity of weighted graph: A new technique to investigate structural complexity of brain activities with applications to aging and autism," Neuroscience letters 650, 103-108.

22. Telesford Q. K., Lynall M. E., Vettel J., Miller M. B., Grafton S. T. and Bassett D. S. 2016, "Detection of functional brain network reconfiguration during taskdriven cognitive states," Neuroimage 142, 188-200.

23. Xie H., Calhoun V. D., Gonzalez-Castillo J., Damaraju E., Miller R., Bandettini P. A. and Mitra S. 2018, "Whole-brain connectivity dynamics reflect both task-specific and individual-specific modulation: A multitask study," Neuroimage 180, 495-504.

24. Dima D. C., Perry G., Messaritaki E., Zhang J. and Singh K. D. 2018, "Spatiotemporal dynamics in human visual cortex rapidly encode the emotional content of faces," Hum Brain Mapp 39, 3993-4006.

25. Heinrichs-Graham E. and Wilson T. W. 2015, "Spatiotemporal oscillatory dynamics during the encoding and maintenance phases of a visual working memory task," Cortex 69, 121-30.

26. Cole M. W., Bassett D. S., Power J. D., Braver T. S. and Petersen S. E. 2014, "Intrinsic and task-evoked network architectures of the human brain," Neuron 83, 238-51.

27. Cole M. W., Ito T., Bassett D. S. and Schultz D. H. 2016, "Activity flow over resting-state networks shapes cognitive task activations," Nat Neurosci 19, 1718-1726.

28. Cheng L., Zhu Y., Sun J., Deng L., He N., Yang Y., Ling H., Ayaz H., Fu Y. and Tong S. 2018, "Principal states of dynamic functional connectivity reveal the link between resting-state and task-state brain: An fmri study," International journal of neural systems 28, 1850002.

29. Demirtaş M., Ponce-Alvarez A., Gilson M., Hagmann P., Mantini D., Betti V., Romani G. L., Friston K., Corbetta M. and Deco G. 2019, "Distinct modes of functional connectivity induced by movie-watching," Neurolmage 184, 335-348.

30. Kauppi J. P., Jaaskelainen I. P., Sams M. and Tohka J. 2010, "Inter-subject correlation of brain hemodynamic responses during watching a movie: localization in space and frequency," Front Neuroinform 4, 5.

31. Regev M., Simony E., Lee K., Tan K. M., Chen J. and Hasson U. 2018, "Propagation of information along the cortical hierarchy as a function of attention while reading and listening to stories," bioRxiv, 291526.

32. Londei A., D'Ausilio A., Basso D., Sestieri C., Gratta C. D., Romani G. L. and Belardinelli M. O. 2010, "Sensory-motor brain network connectivity for speech comprehension," Hum Brain Mapp 31, 567-80.

33. Alavash M., Tune S. and Obleser J. 2019, "Modular reconfiguration of an auditory control brain network supports adaptive listening behavior," Proc Natl Acad Sci U S A 116, 660-669.

34. Broderick M. P., Anderson A. J., Di Liberto G. M., Crosse M. J. and Lalor E. C. 2018, "Electrophysiological correlates of semantic dissimilarity reflect the comprehension of natural, narrative speech," Current Biology 28, 803-809. e3.

35. Hua C., Wang H., Wang H., Lu S., Liu C. and Khalid S. M. 2019, "A Novel Method of Building Functional Brain
Network Using Deep Learning Algorithm with Application in Proficiency Detection," International journal of neural systems 29, 1850015.

36. Engel A. K., Gerloff C., Hilgetag C. C. and Nolte G. 2013, "Intrinsic coupling modes: multiscale interactions in ongoing brain activity," Neuron 80, 867-86.

37. O'Neill G. C., Barratt E. L., Hunt B. A., Tewarie P. K. and Brookes M. J. 2015, "Measuring electrophysiological connectivity by power envelope correlation: a technical review on MEG methods," Phys Med Biol 60, R271-95.

38. O'Neill G. C., Tewarie P., Vidaurre D., Liuzzi L., Woolrich M. W. and Brookes M. J. 2018, "Dynamics of large-scale electrophysiological networks: A technical review," Neuroimage 180, 559-576.

39. Mammone N., De Salvo S., Bonanno L., Ieracitano C., Marino S., Marra A., Bramanti A. and Morabito F. C. 2018 , "Brain Network Analysis of Compressive Sensed HighDensity EEG Signals in AD and MCI Subjects," IEEE Transactions on Industrial Informatics 15, 527-536.

40. Lai M., Demuru M., Hillebrand A. and Fraschini M. 2018 , "A comparison between scalp-and source-reconstructed EEG networks," Scientific reports 8, 12269.

41. Desikan R. S., Ségonne F., Fischl B., Quinn B. T., Dickerson B. C., Blacker D., Buckner R. L., Dale A. M., Maguire R. P. and Hyman B. T. 2006, "An automated labeling system for subdividing the human cerebral cortex on MRI scans into gyral based regions of interest," Neuroimage 31, 968-980.

42. Di Liberto G. M., O’Sullivan J. A. and Lalor E. C. 2015, "Low-frequency cortical entrainment to speech reflects phoneme-level processing," Current Biology 25, 2457 2465.

43. Leonardi N., Richiardi J., Gschwind M., Simioni S., Annoni J. M., Schluep M., Vuilleumier P. and Van De Ville D. 2013, "Principal components of functional connectivity: a new approach to study dynamic brain connectivity during rest," Neuroimage 83, 937-50.

44. Allen E. A., Damaraju E., Plis S. M., Erhardt E. B., Eichele T. and Calhoun V. D. 2014, "Tracking whole-brain connectivity dynamics in the resting state," Cereb Cortex 24, 663-76.

45. Ghosh-Dastidar S., Adeli H. and Dadmehr N. 2008, "Principal component analysis-enhanced cosine radial basis function neural network for robust epilepsy and seizure detection," IEEE Transactions on Biomedical Engineering $\mathbf{5 5}, 512-518$.

46. Nunez P. L. and Srinivasan R. 2006, Electric fields of the brain: the neurophysics of EEG. (Oxford University Press, USA).

47. Delorme A. and Makeig S. 2004, "EEGLAB: an open source toolbox for analysis of single-trial EEG dynamics including independent component analysis," Journal of neuroscience methods $\mathbf{1 3 4}, 9-21$.

48. Cong F., Kalyakin I., Huttunen-Scott T., Li H., Lyytinen H. and Ristaniemi T. 2010, "Single-trial based independent component analysis on mismatch negativity in children," International journal of neural systems 20, 279-292.

49. Rembado I., Castagnola E., Turella L., Ius T., Budai R., Ansaldo A., Angotzi G. N., Debertoldi F., Ricci D. and Skrap M. 2017, "Independent component decomposition of human somatosensory evoked potentials recorded by 
micro-electrocorticography," International journal of neural systems 27, 1650052.

50. Tadel F., Baillet S., Mosher J. C., Pantazis D. and Leahy R. M. 2011, "Brainstorm: a user-friendly application for MEG/EEG analysis," Computational intelligence and neuroscience 2011, 8.

51. Gramfort A., Papadopoulo T., Olivi E. and Clerc M. 2010, "OpenMEEG: opensource software for quasistatic bioelectromagnetics," Biomedical engineering online $\mathbf{9 , 4 5 .}$

52. Hämäläinen M. S. and Ilmoniemi R. J. 1994, "Interpreting magnetic fields of the brain: minimum norm estimates," Medical \& biological engineering \& computing 32, 35-42.

53. Bola M. and Sabel B. A. 2015, "Dynamic reorganization of brain functional networks during cognition," Neuroimage 114, 398-413.

54. Palva S. and Palva J. M. 2012, "Discovering oscillatory interaction networks with M/EEG: challenges and breakthroughs," Trends Cogn Sci 16, 219-30.

55. Lin F. H., Belliveau J. W., Dale A. M. and Hämäläinen M. S. 2006, "Distributed current estimates using cortical orientation constraints," Human brain mapping 27, 1-13.

56. Desikan R. S., Segonne F., Fischl B., Quinn B. T., Dickerson B. C., Blacker D., Buckner R. L., Dale A. M., Maguire R. P., Hyman B. T., Albert M. S. and Killiany R. J. 2006, "An automated labeling system for subdividing the human cerebral cortex on MRI scans into gyral based regions of interest," Neuroimage 31, 968-80.

57. Hipp J. F., Hawellek D. J., Corbetta M., Siegel M. and Engel A. K. 2012, "Large-scale cortical correlation structure of spontaneous oscillatory activity," Nat Neurosci 15, 884-90.

58. O'Neill G. C., Tewarie P. K., Colclough G. L., Gascoyne L. E., Hunt B. A. E., Morris P. G., Woolrich M. W. and Brookes M. J. 2017, "Measurement of dynamic task related functional networks using MEG," Neuroimage 146, 667678.

59. Brookes M. J., Woolrich M. W. and Barnes G. R. 2012, "Measuring functional connectivity in MEG: a multivariate approach insensitive to linear source leakage," Neuroimage 63, 910-20.

60. Colclough G. L., Brookes M. J., Smith S. M. and Woolrich M. W. 2015, "A symmetric multivariate leakage correction for MEG connectomes," Neuroimage 117, 439-48.

61. O’neill G. C., Tewarie P. K., Colclough G. L., Gascoyne L. E., Hunt B. A., Morris P. G., Woolrich M. W. and Brookes M. J. 2017, "Measurement of dynamic task related functional networks using MEG," NeuroImage 146, 667678.

62. Mammone N., De Salvo S., Ieracitano C., Marino S., Marra A., Corallo F. and Morabito F. 2017, "A permutation disalignment index-based complex network approach to evaluate longitudinal changes in brain-electrical connectivity," Entropy 19, 548.

63. O'Neill G. C., Bauer M., Woolrich M. W., Morris P. G., Barnes G. R. and Brookes M. J. 2015, "Dynamic recruitment of resting state sub-networks," Neuroimage 115, 85-95.

64. Kim D., Kay K., Shulman G. L. and Corbetta M. 2017, "A new modular brain organization of the BOLD signal during natural vision," Cerebral Cortex, 1-17.
65. Baldassano C., Chen J., Zadbood A., Pillow J. W., Hasson U. and Norman K. A. 2017, "Discovering Event Structure in Continuous Narrative Perception and Memory," Neuron 95, 709-721 e5.

66. Alday P. M. 2018, "M/EEG analysis of naturalistic stories: a review from speech to language processing," Language, Cognition and Neuroscience, 1-17.

67. Alexandrou A. M., Saarinen T., Kujala J. and Salmelin R. 2018, "Cortical entrainment: what we can learn from studying naturalistic speech perception," Language, Cognition and Neuroscience, 1-13.

68. Sankari Z. and Adeli H. 2011, "Probabilistic neural networks for diagnosis of Alzheimer's disease using conventional and wavelet coherence," Journal of neuroscience methods 197, 165-170.

69. Ahmadlou M., Adeli H. and Adeli A. 2013 , "Spatiotemporal analysis of relative convergence of EEGs reveals differences between brain dynamics of depressive women and men," Clinical EEG and neuroscience 44, 175181.

70. Adeli H., Ghosh-Dastidar S. and Dadmehr N. 2007, "A wavelet-chaos methodology for analysis of EEGs and EEG subbands to detect seizure and epilepsy," IEEE Transactions on Biomedical Engineering 54, 205-211.

71. Sharma P., Khan Y. U., Farooq O., Tripathi M. and Adeli H. 2014, "A wavelet-statistical features approach for nonconvulsive seizure detection," Clinical EEG and neuroscience 45, 274-284.

72. Vidaurre D., Quinn A. J., Baker A. P., Dupret D., TejeroCantero A. and Woolrich M. W. 2016, "Spectrally resolved fast transient brain states in electrophysiological data," Neuroimage 126, 81-95.

73. Palva J. M., Wang S. H., Palva S., Zhigalov A., Monto S., Brookes M. J., Schoffelen J.-M. and Jerbi K. 2018, "Ghost interactions in MEG/EEG source space: A note of caution on inter-areal coupling measures," Neuroimage 173, 632643.

74. Mammone N., Ieracitano C., Adeli H., Bramanti A. and Morabito F. C. 2018, "Permutation Jaccard distance-based hierarchical clustering to estimate EEG network density modifications in MCI subjects," IEEE transactions on neural networks and learning systems 29, 5122-5135. 